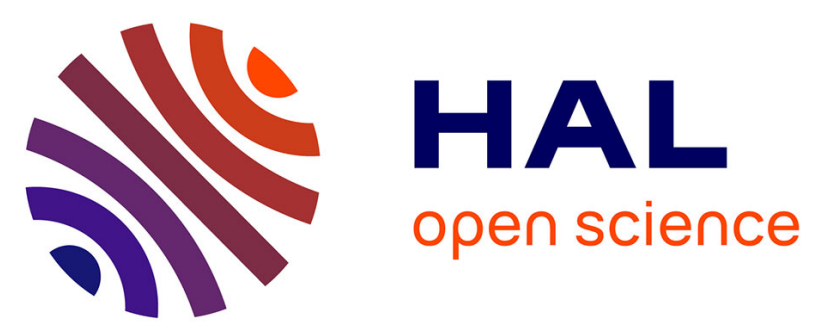

\title{
Stability of vacancy and interstitial dislocation loops in $\alpha$-zirconium: atomistic calculations and continuum modelling
}

Cong Dai, Céline Varvenne, Peyman Saidi, Zhongwen Yao, Mark R Daymond, Laurent Karim Béland

\section{To cite this version:}

Cong Dai, Céline Varvenne, Peyman Saidi, Zhongwen Yao, Mark R Daymond, et al.. Stability of vacancy and interstitial dislocation loops in $\alpha$-zirconium: atomistic calculations and continuum modelling. Journal of Nuclear Materials, 2021, 554, pp.153059. 10.1016/j.jnucmat.2021.153059 . hal03576208

\author{
HAL Id: hal-03576208 \\ https://hal.science/hal-03576208
}

Submitted on 15 Feb 2022

HAL is a multi-disciplinary open access archive for the deposit and dissemination of scientific research documents, whether they are published or not. The documents may come from teaching and research institutions in France or abroad, or from public or private research centers.
L'archive ouverte pluridisciplinaire HAL, est destinée au dépôt et à la diffusion de documents scientifiques de niveau recherche, publiés ou non, émanant des établissements d'enseignement et de recherche français ou étrangers, des laboratoires publics ou privés. 


\title{
Stability of vacancy and interstitial dislocation loops in $\alpha$-zirconium: atomistic calculations and continuum modelling
}

\author{
Cong Dai ${ }^{\mathrm{a}, \mathrm{c}, *}$, Céline Varvenne ${ }^{\mathrm{b}}$, Peyman Saidi ${ }^{\mathrm{c}}$, Zhongwen Yao $^{\mathrm{c}}$, Mark R. Daymond ${ }^{\mathrm{c}}$, Laurent Karim \\ Béland ${ }^{\mathrm{c}}$ \\ ${ }^{a}$ Canadian Nuclear Laboratories, Chalk River, ON KOJ 1J0, Canada \\ ${ }^{b}$ CINaM, UMR 7325, Aix-Marseille Universite, CNRS, F13288, Marseille, France \\ ${ }^{c}$ Department of Mechanical and Materials Engineering, Queen's University, Kingston, ON KYL3N6, Canada
}

\begin{abstract}
We combine atomistic calculations and continuum laws to model irradiation-induced vacancy and interstitial dislocation loops in $\alpha$-zirconium. A comprehensive set of Burgers vectors/stacking sequences in the prismatic and basal planes, of sizes accessible to experiments, are studied by Molecular Statics (MS) simulations. Their formation energies and structural details are determined using two different interatomic potentials for $\alpha$-Zr, considering dislocation loops in hexagonal and circular shapes. Molecular Dynamics annealing of dislocation loops then validates the envisioned potential energy landscape. Finally, the continuum modelling hybridly calibrated on MS results and ab initio data indicate that the coexistence of vacancy and interstitial $\langle a\rangle$ loops is supported by stability arguments. We also establish the limitations of such an approach for quantitative predictions.
\end{abstract}

Keywords: Zirconium, Dislocation loops, Atomistic calculations, Line tension models

\section{Introduction}

Dislocation loops are a major type of irradiationinduced defect that affects structural components of nuclear reactors, including those made of $\mathrm{Zr}$ alloys [1, 2, 3. Accumulation of dislocation loops is a cause of materials' degradation, such as irradiationinduced growth and creep [4, 5]. In particular, pressure tubes in CANDU (CANada Deuterium Uranium) reactors elongate at a rate of $3-4 \mathrm{~mm}$ per year [6]. The different irradiation growth regimes proposed by Holt et al. [7] correlate with the appearance of different types of dislocation loops. At low irradiation doses $\left(<3 \times 10^{25}\right.$ neutrons $\left./ \mathrm{m}^{2}\right)$, most dislocation loops are perfect loops, and have a Burgers vector $\langle a\rangle=1 / 3\langle 11 \overline{2} 0\rangle$ [2, 8]. They are both of vacancy and interstitial type [4, 9], and their habit plane is close to the first-order prismatic $\{10 \overline{1} 0\}$ planes, with a small misorientation [2, 10]. At higher irradiation doses $\left(>6 \times 10^{25}\right.$ neutrons $/ \mathrm{m}^{2}$ ), vacancy-type loops lying in the basal

\footnotetext{
* Corresponding author

Email address: cong.dai@cnl.ca (Cong Dai)
}

plane appear, which may be responsible for accelerated irradiation growth [1]. These loops have a $\langle c\rangle$ component Burgers vector of $1 / 6\langle 20 \overline{2} 3\rangle$, and are faulted, as observed by fringe contrast in transmission electron microscopy (TEM) [11, [12, 13. Note finally that Griffiths et al. [14] observed both vacancy and interstitial $\langle c\rangle$ component loops after electron irradiation; Interstitial $\langle c\rangle$ component loops have not yet been reported in neutronirradiated samples.

The proportion of vacancy to interstitial $\langle a\rangle$ loops is controlled in part by temperature. Indeed, experiments [2, 13, 15, 16] reveal that interstitial loops predominate below $573 \mathrm{~K}$; the number of vacancy and interstitial loops is close between $573 \mathrm{~K}$ to $673 \mathrm{~K}$, while vacancy loops predominate above $673 \mathrm{~K}$. Following neutron irradiation, Jostsons et al. 10] reported that large vacancy $\langle a\rangle$ type loops of diameter $>40 \mathrm{~nm}$ are mostly elliptical, while small loops with diameter $<40 \mathrm{~nm}$ are circular, in agreement with Ref. [17. They also reported that $\langle a\rangle$ interstitial loops are more circular. $\langle a\rangle$-type loop diameter ranges from $5-150 \mathrm{~nm}$ 2. 13, 15, 18. On the other hand, the density

April 8, 2021 
of $\langle c\rangle$-type loops is much lower than that of $\langle a\rangle$ loops, but their diameter is much larger, ranging from $50-200 \mathrm{~nm}$, as observed by TEM [10, 18, 19]. A few experimental works on Zircaloy-2 recently explored their shape. Atom probe tomography (APT) of neutron-irradiated samples 20 showed segregated Fe to "ring-shaped" features interpreted as $\langle c\rangle$-component loops; a TEM work from Harte et al. 18 found circular $\langle c\rangle$-component loops after proton-irradiation.

In summary, there is a link between the irradiation growth behavior and the different types of dislocation loops. A good understanding of the formation and growth mechanisms of the different loops versus irradiation dose is thus needed, so as to improve the predictive capabilities of the models for irradiation-induced growth, and discriminate between the possible scenarios [21, 22, 23, 24]. This includes in particular (i) improving the knowledge of the microstructural details of the irradiation extended defects, and (ii) getting more quantitative information on both thermodynamic and kinetic aspects, e.g. on the relative stability of the various extended defects, and on the elementary steps responsible for their growth, mobility, etc.

Theoretical and simulation progress has been made recently on the topic. On the thermodynamic side, continuum models to predict the vacancy loop's formation energy have been proposed in Refs. [25, 26]; they describe the total energy of the defect as a sum of a line tension term, a stacking fault energy term, and possibly a constant term, following Ref. 27. Such a modelling approach was validated using Molecular Statics (MS) simulations based on an embedded atom method (EAM) potential by Mendelev and Ackland (labelled \#2) [28, performed for all possible types of vacancy loops having hexagonal shape and various possible stacking faults. Then, these laws were calibrated using density functional theory (DFT) data and used to predict the formation energies of loops up to sizes easily observable by TEM. Perfect $\langle a\rangle$ vacancy loops were predicted to be energetically favored over $\langle c\rangle$ loops, in agreement with the experimental observation of predominantly $\langle a\rangle$ loops at lower fluences. On the elementary processes side, a mechanism by which hexagonal $\langle c\rangle$-component vacancy loops can form has been proposed in two recent works [29, 26], where vacancy-type stacking fault pyramids - having hexagonal base and lying in the basal plane - are suggested as precursors for the formation of $\langle c\rangle$ loops. Dai et al. 29] established, through a combination of TEM and molecular dynamics (MD) simulations with the EAM \#3 potential [28], that small vacancy stacking fault (SF) pyramids can form from the interaction between collision cascades and a dense cluster of pre-existing $\langle a\rangle$-type vacancy loops randomly distributed on a common basal plane. Christiaen et al. [26, using $a b$ initio calculations of $\langle c\rangle$-type and $\langle c\rangle$-component vacancy loops energetics at various defects sizes, showed that $\langle c\rangle$ loops are unstable towards these stacking fault pyramids at small sizes. As the loop size increases, ab initio-informed continuum modeling and EAM \#3 simulations demonstrated the expected $\langle c\rangle$-loops to be stable and energetically more favorable.

On the other hand, fewer atomistic simulations have been performed on the stability of interstitial loops in $\alpha-\mathrm{Zr}$, and no continuum models have been used to describe them at large scale. Diego et al. [30, 31] determined the energetics of various interstitial clusters (up to 300 point defects) in the second-order prismatic and basal planes, using the EAM potential by Ackland 32 and the EAM \#3 potential. Following a bottom-up approach by using the single SIA configurations having the lowest energy as building-blocks, they created the dislocation loops. The authors found the energetics of these interstitial loops to be rather close to those of vacancy loops having the same Burgers vectors. However, the SIA configurations used are not always the most stable ones, as predicted by ab initio calculations 33, 34, 35, and/or the obtained dislocation loops do not match the experimental observations (in terms of habit planes, stacking sequence or loop shape). Since the existence of interstitial loops was proved by a number of previous experiments [2, 13, 15, 17, 18, and in order to understand the coexistence of vacancy and interstitial $\langle a\rangle$ loops, while most $\langle c\rangle$ loops are of vacancy-type, a detailed study of interstitial loops is of importance.

The present work therefore aims at investigating the energetics and structure of extended defects having both vacancy and interstitial character, at sizes larger than those studied in previous works, and not readily accessible via $a b$ initio calculations. This allows us to consider only defects whose structure corresponds to fully collapsed dislocation loops, and to avoid the small defect size zone that involves complex configurations [36, 37. The popular EAM \#2 and \#3 potentials 28. for hcp Zr are selected, and MS calculations are carried 
out for dislocation loops of increasing size, having the desired vacancy or interstitial type and Burgers vector/stacking sequence, and with fixed initial hexagonal or circular shapes. Next, MD annealing simulations are performed to further relax the dislocation loops, and their structure and energetics are compared to those obtained by MS simulations. Then, a continuum line-tension model is adjusted on the atomistic results in order to (i) test their ability to capture the differences between vacancy and interstitial loops, (ii) possibly quantify the effect of the loop shape, and (iii) extrapolate the formation energies at loop sizes comparable to those observe experimentally, using an ab initio parameterization. Note that these continuum laws can be subsequently used as inputs to mesoscale numerical approaches to defect evolution under irradiation (Cluster Dynamics, Object Kinetic Monte Carlo, etc.). We finally discuss the limitations of such a simplified approach for quantitative predictions.

\section{Methods and dislocation loop details}

\subsection{Atomistic simulations and defect creation}

Our atomistic simulations are performed using the open-source code LAMMPS (Large-scale Atomic/Molecular Massively Parallel Simulator) 47, using the two EAM potentials for $\mathrm{Zr}$ developed by Mendelev and Ackland and denoted as \#2 and \#3 in Ref. 28]. Their description of hcp Zr bulk properties, i.e. lattice parameters and elastic constants, are both reasonable. The EAM \#2 potential provides a better description of vacancy clustering at small defect sizes than the EAM \#3 potential-as compared to ab initio calculations-and does not present the nonphysical structural stabilization of the $B B$ stacking along the [0001] direction that exists with the EAM \#3 potential 25. On the other hand, the EAM \#3, that was developed for plasticity purposes, is better in describing the different stable stacking fault energies of hcp Zr. In particular, the ordering of the various basal and prismatic faults is similar to the ab initio predictions [42, 25, 44, as can be seen in Table 1. The EAM \#3 potential is also able to predict the existence of stable stacking fault pyramids, a vacancy cluster-type that was recently identified by $a b$ initio calculations [26]. Neither of these two interatomic potentials is perfect, but they have complementary characteristics for studying vacancy and self-interstitial dislocation loops in hcp Zr. Comparing them will be helpful in identifying which features of the loops are generalizable - and transposable to an ab initio informed continuum model - and which features are potential-dependent - and therefore not transposable.

MS simulations, i.e. energy minimization at $T=0 \mathrm{~K}$, are performed using a conjugate gradient algorithm, and imposing zero pressure on the simulation boxes. Dislocation loops are created using the BABEL package [48, with the procedure detailed in Ref. 25] and summarized here. A platelet of vacancies/SIAs is first removed/added into the hcp Zr matrix; then, using Burgers formula for dislocation loops 49, the plastic part of the displacement field is applied to the atoms in the supercell. This allows us to introduce a priori the desired dislocation loop, having the targeted stacking fault, through the choice of the Burgers vector. Various shapes based on triangular elementary loops can be generated with this software: here, both hexagonal and almost circular loops were considered. Periodic boundary conditions were applied to all directions, and the total number of atoms is 2.3 million in these MS simulations. This supercell size was large enough to ensure well converged defect structures and energies for our maximum loop size of $\sim 1135$ point defects (see Appendix A for details).

MD simulations were performed within the isobaric-isothermal $(N, p, T)$ ensemble, starting with an annealing at $573 \mathrm{~K}$ for at least $500 \mathrm{ps}$ and followed by a cooling down to $3 \mathrm{~K}$ over at least $100 \mathrm{ps}$. An energy minimization was finally performed using the conjugate gradient algorithm to calculate the potential energy of dislocation loops. Only circular loops were created in this case, following the method outlined in Ref [50, that consists in a simple removal/addition of a cylinder of $\mathrm{Zr}$ atoms in the desired habit plane. With this procedure, the obtained dislocation loop stacking sequence-either perfect or with a specific stacking fault-then results from the various energy barriers that are overcome during the MD annealing. The total number of atoms in MD simulations is 4 million.

Atomic configurations of the vacancy and interstitial defects were visualized and analyzed with the Open Visualization Tool [51. Crystal structures are identified using common neighbor analysis (CNA) [52] and the dislocation extraction algorithm (DXA) 53. The Burgers vector of some dislocation segments that DXA could not properly label-namely $\langle c\rangle$-component loops of Burgers vector $1 / 6\langle 20 \overline{2} 3\rangle$-were calculated by a homebrewed Matlab script, described in Ref. 50]. 
Table 1: Lattice parameters $a$ and $c$, elastic constants $C_{i j}$ and stacking fault energies $\gamma_{10 \overline{1} 0}, \gamma_{E}, \gamma_{I_{1}}, \gamma_{I_{2}}, \gamma_{\Pi_{1 \mathrm{~L}}}$ and $\gamma_{\Pi_{1 \mathrm{D}}}$ of hcp Zr. The values for the EAM potentials \#2 and \#3, are obtained from Refs. 25, 28, 38, 39, 40, and from this work for $\gamma_{\Pi_{1 D}}$ with EAM \#2, following the method of Ref. 39. Ab initio values correspond to calculations using the PBE 41 functional. Ultra-soft pseudopotential (US) calculations are from Refs. 25, 42, 39, 43, and includes 12 electrons in valence, and plane augmented wave (PAW) calculations are from Ref. [4].

\begin{tabular}{lccccccccccccc}
\hline Method & $a(\AA)$ & $c / a$ & \multicolumn{10}{c}{ Elastic constants $(\mathrm{GPa})$} & \multicolumn{1}{c}{ Fault energies $\left(\mathrm{mJ} / \mathrm{m}^{2}\right)$} \\
\cline { 4 - 14 } & & & $C_{11}$ & $C_{33}$ & $C_{12}$ & $C_{13}$ & $C_{44}$ & $\gamma_{1010}$ & $\gamma_{E}$ & $\gamma_{I_{1}}$ & $\gamma_{I_{2}}$ & $\gamma_{\Pi_{1 \mathrm{~L}}}$ & $\gamma_{\Pi_{1 \mathrm{D}}}$ \\
\hline EAM \#2 & 3.220 & 1.619 & 149 & 180 & 82 & 65 & 48 & 357 & 164 & 55 & 110 & 278 & 268 \\
EAM \#3 & 3.234 & 1.598 & 142 & 168 & 75 & 76 & 44 & 135 & 297 & 99 & 198 & 136 & 243 \\
PWSCF US 12e & 3.230 & 1.601 & 140 & 168 & 70 & 65 & 26 & 211 & 274 & 147 & 213 & 127 & 163 \\
VASP PAW & 3.235 & 1.598 & 147 & 164 & 70 & 71 & 25 & 183 & 284 & 149 & 224 & 103 & - \\
Exp. 45, 46] & 3.232 & 1.603 & 155 & 172 & 67 & 65 & 36 & & & & & \\
\hline
\end{tabular}

\subsection{Investigated dislocation loops}

(a) prism perfect

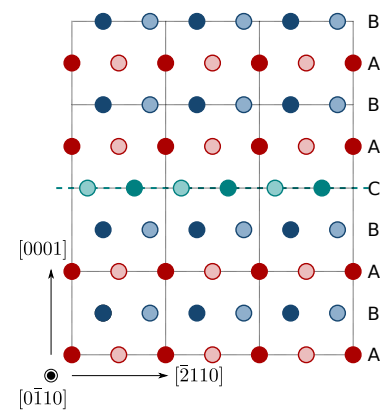

(c) basal E

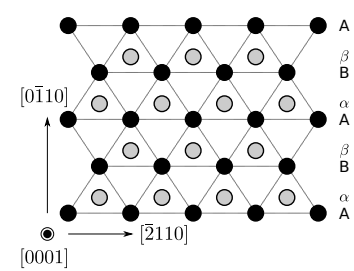

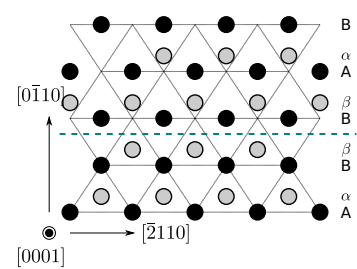

(b) prism faulted

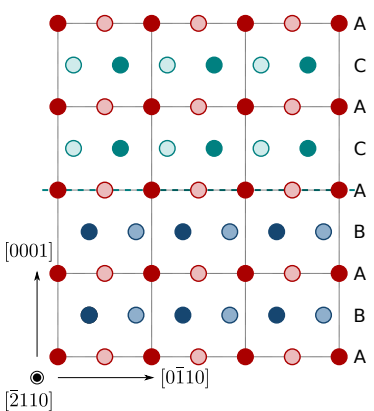

(d) basal $I_{1}$
Figure 1: Stacking sequences normal to loop planes encountered in (a-b) prismatic loops, and in (c-d) basal loops. Atoms are sketched either by filled or open symbols, depending on their position in the plane normal to the picture. In (c-d), atoms are colored in red, blue and green to represent $\mathrm{A}, \mathrm{B}$ and $\mathrm{C}$ atomic planes along the [0001] direction. Dashed lines indicate the stacking fault planes.

We detail here the different dislocation loops that were created and examined in this work. For a pedagogic description of the different possible stacking faults associated with the addition/removal of matter in hcp metals, the reader is referred to Ref. 54.

Two dislocation loops lying in the basal plane and having different stacking faults are investigated in the present study. First, dislocation loops of Burgers vector $\vec{b}_{1}=1 / 2[0001]$ and having an extrinsic $\mathrm{E}$ fault are considered, their stacking sequence along the [0001] direction is $A B A B C A B A B$ (see Figure 1(d)) instead of $A B A B A B A B$. Second, dislocation loops with Burgers vector $\vec{b}_{2}=1 / 6\langle 20 \overline{2} 3\rangle$ and an intrinsic fault $\mathrm{I}_{1}$ leading to a stacking sequence of $A B A B C B C B$ along the [0001] direction (Figure 1(c)), are studied. The formation of basal vacancy (resp. interstitial) loops are described in textbooks 54 as the condensation of vacancies (resp. SIAs) onto a basal plane followed by the introduction of an $\mathrm{E}$ or $\mathrm{I}_{1}$ fault to lower energy. The vacancy type of these intrinsic $I_{1}$ faulted loops correspond to the widely observed $\langle c\rangle$-component loops in irradiated Zr.

In the prismatic $\{10 \overline{1} 0\}$ planes, again two different kinds of loops can be found: faulted dislocation loops with Burgers vector $\vec{b}_{3}=1 / 2\langle 10 \overline{1} 0\rangle$, and perfect (or unfaulted) prismatic loops with Burgers vector $\vec{b}_{4}=1 / 3\langle 2 \overline{1} \overline{1} 0\rangle$. Their respective stacking sequence in the normal loop plane direction is shown in Figure 1(a) and (b).

These four types of loops are studied in this work, for both vacancy and interstitial character. Both hexagonal and circular shapes are selected: the former (i) to check consistency with previous atomistic works on vacancy loops 25, 26 and (ii) because hexagonal vacancy $\langle c\rangle$ loops appear beyond a critical size by transformation of SF pyramid vacancy defects 29, 26, and the later because this shape is usually seen in experiments. 


\section{Results of Molecular Statics simulations}

In this section, the structure and energetics of vacancy and interstitial dislocation loops in hcp $\mathrm{Zr}$ are presented. The formation energy $E_{f}(n)$ of a dislocation loop consisting of $n$ point defects introduced in a simulation box that initially contains $N$ bulk atoms is defined as:

$$
E_{f}(n)=E_{p}^{\text {loop }}(n)-\frac{N \pm n}{N} E_{p}^{\text {bulk }},
$$

where $E_{p}^{\text {loop }}(n)$ and $E_{p}^{\text {bulk }}$ are the potential energies of the same simulation cell containing one vacancy/SIA loop and a perfect crystal, respectively. $N \pm n$ represents the total number of atoms in a system with an interstitial and a vacancy loop, respectively. Dislocation loops of 8 different sizes are investigated, ranging from $\sim 4 \mathrm{~nm}$ to $\sim 11 \mathrm{~nm}$ in diameter, corresponding to 127 to 1135 point defect: 17 . These intermediate sizes correspond to a range where the defects have a fully collapsed dislocation loop structure, and though of a few nm, fall into the typical size range that can be observed by TEM.

\subsection{Energetics of vacancy dislocation loops}

The MS formation energies for hexagonal and circular vacancy loops of intermediate size range, obtained after MS relaxation using the EAM \#2 and \#3 potentials, are plotted versus the number of vacancies in Figure 2(a) and (b), respectively.

Our atomistic results are consistent with the published literature, obtained for the same loop-types having up to $\sim 300$ vacancies with the EAM \#2 potential [25], and for $\langle c\rangle$-component loops having up to $\sim 4000$ vacancies with the EAM \#3 potential [26]. The initially introduced stacking sequences are essentially preserved during the static relaxation (see Supplementary Information for complete atomistic views). Two exceptions are found at small sizes: the perfect prismatic loops, that are unstable towards faulted prismatic loops for both potentials, and the $I_{1}$ basal loops with the EAM \#3 potential, that are unstable towards a $B B$ stacking sequence, which is known to be artificially metastable with this interaction model [42, 25]. These data points are thus removed from the formation energy curves of Figs. 2(a) and (b).

\footnotetext{
${ }^{1}$ The link between the radius of a dislocation loop and the number of point defects $n$ constituting the loop is given in Ref. 25].
}

In general the formation energy values are larger with the EAM \#2 potential, which agrees with its larger vacancy binding energy as compared to the EAM \#3 potential 25]. Both potentials predict a formation energy for the prismatic loops lower than that for the basal loops in the whole range of investigated loop size. Faulted prism loops become less stable than the perfect prism loops with increasing loop size, and the larger the loop area, the larger their energy difference. This energy difference is more pronounced with the EAM \#2 potential, due to its higher $\gamma_{10 \overline{1} 0}$ value. Concerning basal vacancy defects, E-faulted loops are less stable than the $I_{1}$-faulted loops. Their formation energy difference also increases with the loop area, but is more pronounced for the EAM \#3 potential, for which $\gamma_{E}-\gamma_{I_{1}}$ is higher.

Finally, we note that the differences induced by the shape of the loops - here hexagonal and circular shapes are tested - on the vacancy loop formation energies is rather small. This rather limited sensitivity of loop energetics to the loop shape was already shown in earlier empirical potential studies involving vacancy loops of rectangular shapes with different aspect ratios [30]. Here, it is noted for both potentials that after relaxation, small circular basal loops present some facets in the same direction as those of hexagonal loops; this makes the shape variations rather limited between the datasets, thus reducing the differences in formation energies.

\subsection{Energetics of interstitial dislocation loops}

We now consider MS calculations for the same types of loops but having interstitial character. Results for hexagonal and circular loops' formation energies are displayed in Figure 3(a) and (b), and the atomistic top views of hexagonal loops after MS relaxation are given in Figure 3(c) and (d), for EAM $\# 2$ and \#3, respectively.

The stacking sequences of the hexagonal loops are identical to those initially introduced for the perfect $\langle a\rangle$ loops, and for E-faulted and $\mathrm{I}_{1}$-faulted basal loops, whatever the defect size within the investigated range. Contrary to the case of vacancy loops, faulted $\langle a\rangle$ loops are unstable towards perfect $\langle a\rangle$ loops from loops having 300 SIAs with EAM $\# 2$, and unstable towards partially unfaulted loops at all sizes with EAM \#3. For the former potential, we note that the loop unfaulting is only partial after relaxation with the usual CG algorithm, and that total unfaulting arises when using the robust steepest descent minimization algorithm. This total un- 

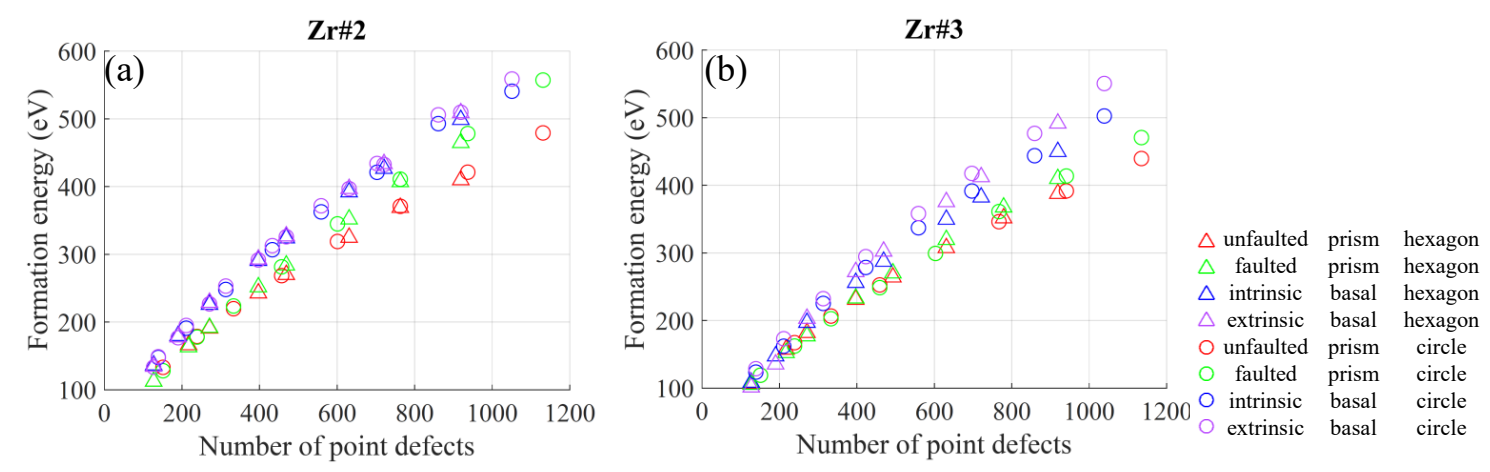

Figure 2: Formation energies of vacancy loops calculated by the Zr \#2 (a) and the \#3 (b) potentials, using Molecular Statics (MS). Hexagonal and circular loops are represented by triangles and circles, respectively. Four different types of vacancy loops were calculated and they are in different colors (for interpretation of the references to color in this figure legend, the readers is referred to the web version of this article).

faulting does not occur with the EAM \#3. Those unstable configurations are thus removed from the faulted $\langle a\rangle$-loop dataset in Figure 3 for the sake of clarity.

For both $\mathrm{Zr}$ potentials, the formation energies of hexagonal basal SIAs loops are generally higher than that of prism SIAs loops. The formation energies of SIAs loops predicted by the \#2 potential are typically higher than those predicted by the \#3 potential. As for vacancy loops, the formation energy difference between the intrinsic and extrinsic basal SIAs loops predicted by the \#2 potential is smaller than that predicted by the \#3 potential; it increases with the loop area, which can again be related to the difference in the $\gamma_{E}-\gamma_{I_{1}}$ quantity existing between the two potentials. Comparing now the formation energy values of hexagonal SIAs loops to their vacancy loop counterparts, it is found for both potentials that they are comparable for the $\mathrm{I}_{1}$-faulted basal loops, and that vacancy Efaulted loops have higher formation energies than the SIAs ones, the difference being more important with EAM \#2. For perfect prismatic loops, however, the stability ordering is potential-dependent: SIAs loops are less stable than vacancy loops with EAM \#2, while SIAs loops are more stable than vacancy loops with EAM \#3. This could be connected with the dislocation loop structural details that are discussed in the next section.

We finally discuss on the results for circular SIAs loops (see atomistic views in Supplementary Information for details). Their stacking sequences are identical to the hexagonal loops of comparable size, except for the faulted $\langle a\rangle$-loops with the EAM \#3 potential. Some of the circular faulted loops are stabilized and others unstable towards totally or partially faulted loops (see related formation energy data-points in Fig. 3(b)), while all hexagonal faulted loops are unstable. This could also be related to the details of the energy minimization. As for vacancy loops, the energetic differences between circular and hexagonal SIA loops are not very pronounced, and are in general lower than those observed between vacancy and interstitial loops. Most circular prismatic loops remain circular after the relaxation, but small circular basal loops spontaneously become faceted after relaxation with both potentials, indicating that there is a partial shape instability towards hexagonal loops at small sizes. This is similar to our observations for circular vacancy basal loops, and agrees with the very similar formation energies of circular and hexagonal loops.

\subsection{Structural analysis of the loop segments}

We now pay attention to the structural details of the dislocation segments forming the loops, as those can have important consequences for (i) the mobility of the loops and (ii) the applicability of the continuum modelling of dislocation loop energetics, as will be seen in section 5 . To this aim, we focus on the largest hexagonal loops, that contain 919 point defects, and comment on the dislocation loop size and shape effects when useful. Focusing on hexagonal loops is in part motivated by the theoretical prediction of hexagonal vacancy basal loops by transformation of SF pyramidal vacancy defects [26, 29]. Figure 4 provides perspective views of the vacancy 


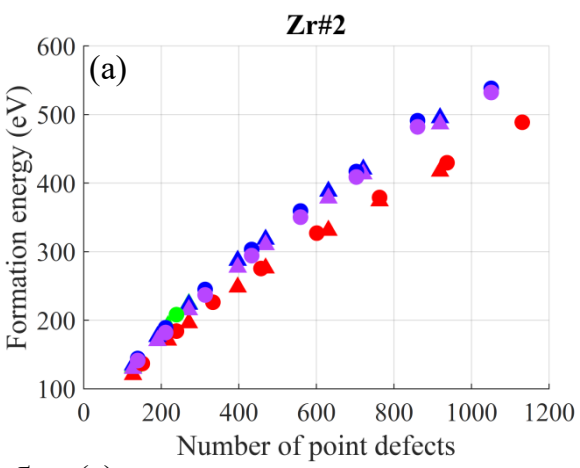

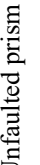
(c)

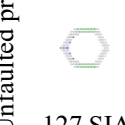

27 SIAs 271 SIAs

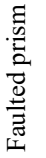

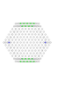

127 SIAs

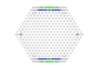

271 SIAs

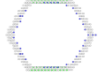

469 SIA

$\overline{0}$
0
0
0
0
0
0

0
0
0
0
0
0
0
0
0
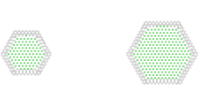

27 SIAs

271 SIAs

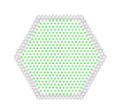

469 SIAs

$\vec{w}$
$\tilde{0}$
0
0
0
0
0

0
0
0
0
0
0

新 127 SIAs

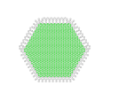

271 SIAs

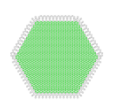

469 SIAs
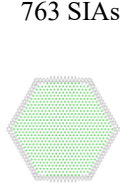

721 SIAs

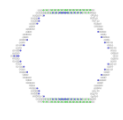

763 SIAs
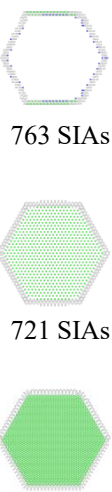

721 SIAs
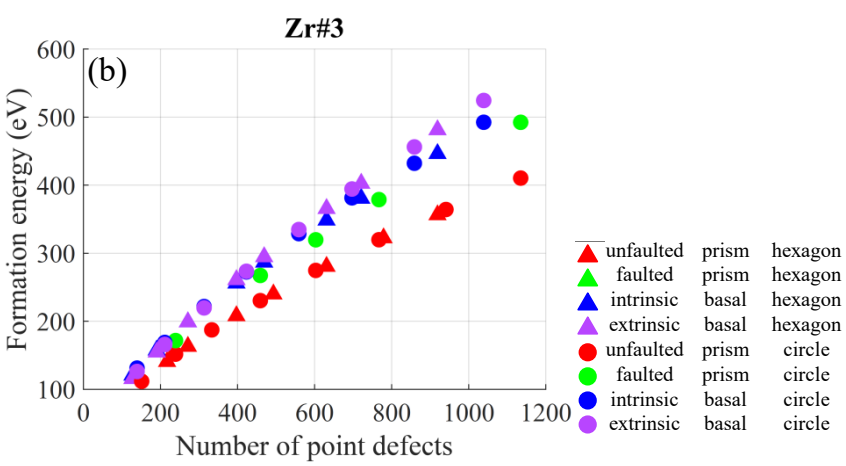

(d)

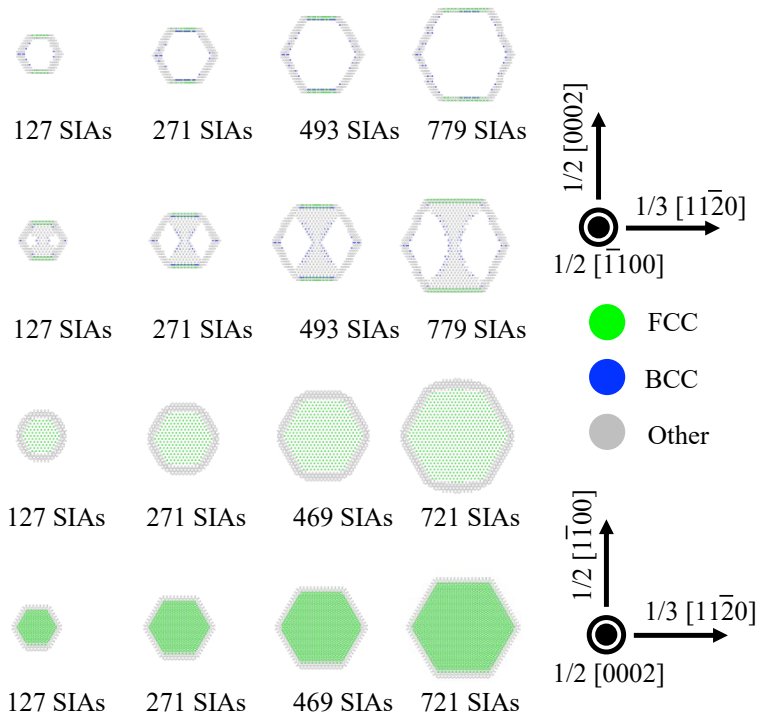

Figure 3: Results of MS calculations for SIAs dislocation loops. (a) and (b): formation energies of interstitial loops calculated by the Zr EAM \#2 (a) and the \#3 (b) potentials. Hexagonal and circular loops are represented by triangles and circles, respectively, and colors distinguish the kind of loop. (c) and (d): atomistic top views of the hexagonal SIAs loops after MS relaxation with the EAM \#2 and \#3 potentials. Atoms are colored according to the CNA, i.e. green/blue is for fcc/bcc local environment and gray for undetermined environment.

and SIAs prismatic and basal loops, after MS relaxation using the EAM \#2 and \#3 potentials (see also Supplementary Information for atomistic views of other loop sizes and of circular loops). Faulted prismatic loops, since they are unstable with both potentials at large size, are not discussed here.

\subsubsection{Prismatic perfect loops}

We first consider prismatic loops having Burgers vectors $\vec{b}_{4}=1 / 3\langle 2 \overline{1} \overline{1} 0\rangle$. Performing the DXA after the static relaxation allows for the analysis of the various loop segments. As can be seen on Figure 4 . the habit planes of the loops are slightly tilted with respect to the initial prismatic plane, with the tilt-
Table 2: Dissociation distances $d_{\text {eq }}$ in $\AA$, measured in atomistic simulations or predicted by anisotropic elasticity using the relaxed line directions, for the prismatic loop segments lying either in the basal or in the pyramidal plane. Both atomistic and theoretical values are averaged over the two (resp. four) segments of each loop lying in similar planes.

\begin{tabular}{llcccc}
\hline & & \multicolumn{2}{c}{ EAM \#2 } & \multicolumn{2}{c}{ EAM \#3 } \\
\cline { 3 - 6 } Segment & Loop & Sim. & Elast. & Sim. & Elast. \\
\hline Basal & V & 16.30 & 17.09 & 11.15 & 8.33 \\
& SIAs & 16.95 & 17.20 & 12.07 & 9.34 \\
Pyr. $\Pi_{1 D}$ & V & - & 5.06 & - & 5.75 \\
& SIAs & - & 5.05 & - & 5.99 \\
\hline
\end{tabular}




\begin{tabular}{|c|c|c|c|c|c|}
\hline & \multicolumn{2}{|c|}{$\mathrm{Zr} \# 2$} & \multicolumn{2}{|c|}{$\mathrm{Zr} \# 3$} & FCC \\
\hline & Vacancy & SIAs & Vacancy & SIAs & Other \\
\hline 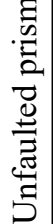 & & & & & 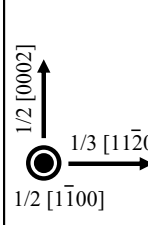 \\
\hline 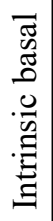 & & & & & \\
\hline 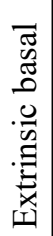 & & & & & \\
\hline
\end{tabular}

Figure 4: Atomistic configurations of unfaulted prism, intrinsic and extrinsic basal loops in both vacancy and SIAs type obtained by the Zr \#2 and the \#3 potentials using MS. The loops are in hexagonal shape with 919 point defects.

ing angle depending both on the potential and on the vacancy or interstitial character of loop.

For the two dislocation segments lying in the basal plane, a dissociation is observed, corresponding to

$$
\frac{1}{3}\langle 1 \overline{2} 10\rangle \rightarrow \frac{1}{3}\langle 1 \overline{1} 00\rangle+\frac{1}{3}\langle 0 \overline{1} 10\rangle,
$$

with the two partials separated by a basal $\mathrm{I}_{2}$ stacking fault. This basal dissociation was also observed by de Diego et al. [30, 31] with the EAM \#3 potential, on rectangular vacancy and SIAs prismatic loops. The atomistically-measured dissociation distances in the basal plane are given in table 2 , measurement are done by using the DXA and by averaging between the two basal segments of each dislocation loop. Dissociation is more important for the interstitial loops than for the vacancy loops for both potentials, and the EAM \#3 leads to shorter dis- sociations than the EAM \#2. To rationalize these results, and as we are considering rather large dislocation loops, we make use of the anisotropic theory of elasticity for infinite straight dislocations [49] (see Appendix C) to predict the dissociation distances $d_{\text {eq }}$ of each prismatic loop. To do so, we use the Burgers vectors, elastic constants and relevant stacking fault energies given in Table 1 for the two interaction models, and the line/segment directions extracted from simulations with the DXA. The obtained $d_{\text {eq }}$ values are displayed in Table 2. The agreement of the elasticity predictions with atomistics is fairly good: specific values are close, and the larger dissociations are observed for the EAM \#2 potential and for the SIAs type of loops. In particular, the different dissociation length observed for vacancy and SIAs types of loops can be ascribed to the difference in dislocation segment orientation 
after MS relaxation. Indeed, with their initial orientation, a similar dissociation distance would have been predicted for both vacancy and SIAs types. The relaxation slightly changes each loop character, which finally results in varying $d_{\text {eq }}$.

The four remaining segments forming the hexagonal perfect prismatic loops show some spreading in pyramidal dense planes $\Pi_{1 D}$ (see Ref. [40] for details). Inspection of the non-hcp atoms after CNA in simulations indicates a dislocation spreading that is a bit larger with the EAM \#3 potential. With both potentials, the spreading is slightly larger for the segments of the SIAs loops. Again, predictions of $d_{\text {eq }}$ are performed using elasticity theory. The considered dissociation is described as

$$
\begin{aligned}
\frac{1}{3}\langle 11 \overline{2} 0\rangle \rightarrow & \frac{1}{6}\langle 11 \overline{2} 0\rangle+\alpha\langle\overline{1} 102\rangle \\
& +\frac{1}{6}\langle 11 \overline{2} 0\rangle-\alpha\langle\overline{1} 102\rangle,
\end{aligned}
$$

with $\alpha$ a parameter whose value depends on the interaction model [39, 40. The obtained $d_{\text {eq }}$ values are provided in Table 2. They are smaller than those for basal dislocation segments; they correspond to a spreading instead of well separated partials, and are in reasonable agreement with the qualitative atomistic observations.

The 919 point defects (PDs) hexagonal loops are large but finite objects: interactions between the straight dislocation segments and an influence of the loop edges could exist. The rather good agreement with the elasticity predictions for infinite straight dislocations thus indicates that those effects are not dominant for this loop size. The $a b$ initio values of $\gamma_{\mathrm{I}_{2}}$ and $\gamma_{1 D}$ (see Table 1), suggest that the presently observed core structures of hexagonal prism loop segments are plausible ${ }^{2}$. Besides, the above analysis shows a coupling between the dissociation of the loop segments and the final orientation of the loop habit planes. Indeed, the ability of the loop segments to dissociate facilitates their subsequent glide, which then allows the loop to reorientate. This determines the relaxed dislocation segment character and fixes the equilibrium dissociation distance of each segment. This effect is dependent on the size and shape of the dislocation loop. In particular, both habit plane orientation and dissociation lengths vary with loop

\footnotetext{
${ }^{2}$ Basal dissociation is unstable for infinite screw dislocations in $a b$ initio calculations [42; but such dissociation could occur for finite connected segments of mixed character.
}

size (see Appendix B); they converge towards fixed values for loops $\gtrsim 800$ point defects. Inspection of circular loops shows very small misorientation angles as compared to hexagonal loops. Concomitantly, we observe more limited dissociation of dislocation portions, likely prevented by the significant curvature of this loop shape. Therefore, the loop shape, while having a modest effect on the formation energy, is important for the segment dissociation, and then for the local mobility/reorientation of the loop, which could possibly influence (i) its subsequent long range mobility 55, 56 and (ii) its capture cross-section for diffusing point defects.

\subsubsection{Basal loops}

With the EAM \#3 potential, extrinsic and intrinsic basal loops of both vacancy and SIAs types shows rather large areas of first order pyramidal loose plane $\Pi_{1 \mathrm{~L}}$ [40]. These stacking fault portions are oriented inward the hexagon for the vacancy loops, and outward for the SIAs loops. As seen in Figure 4, the pyramidal stacking fault area is more important for vacancy (resp. $\mathrm{I}_{1}$-faulted) basal loops as compared to SIAs (resp. E-faulted) basal loops. The development of these faults has already been identified in several works on vacancy loops [26, 23, and is likely reminiscent from the SF pyramid-type of defect that is more stable than simple vacancy loops at small defect sizes. In agreement with this, the stacking fault areas are more pronounced in small loops.

On the other hand, with the EAM \#2 potential, the development of areas of pyramidal $\Pi_{1 \mathrm{~L}}$ stacking faults is not observed; at most some core spreading is seen for the dislocation segments. This is well explained by the much higher value of $\gamma_{\Pi_{1 L}}$ for this potential, that is also higher than the $a b$ initio value. All basal loop habit planes are essentially not tilted with respect to the initial basal plane after MS relaxation.

Interestingly, all circular basal loops having sizes up to $\sim 919$ point defects evolve towards more hexagonal shapes during the MS relaxation (see Supp. Info.). The dissociation and/or core spreading in the pyramidal planes of the dislocation loop segments could be the driving force for this. A few experiments [18, 20] mentioned that the shape of $\langle c\rangle$-component loops was circular. However, a careful examination of a TEM image (Fig.4(c) of Ref. [18] ) shows some faceting of $\langle c\rangle$-component vacancy loops; those of diameter $\sim 50 \mathrm{~nm}$ almost appear as hexagons. A hexagonal shape is observed 

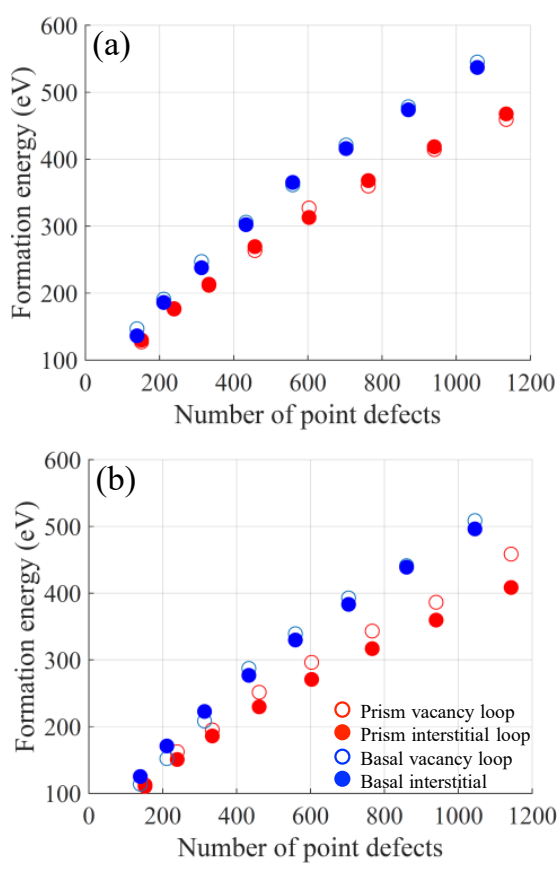

Figure 5: Formation energies of loops calculated by the $\mathrm{Zr}$ \#2 (a) and the $\mathrm{Zr}$ \#3 (b) potentials after MD relaxation. Basal loops are colored in red, and prismatic loops in blue. Open (reps. filled) symbols corresponds to vacancy (resp. interstitial) defects.

indirectly by APT for loop diameters $\sim 40 \mathrm{~nm}$, through the segregation of Fe atoms onto loop segments (Fig. 8 of Ref. 20]). In agreement with this, the present result of shape instability - together with the atomistic and $a b$ initio predictions of SF pyramids at small vacancy defect sizes - suggests hexagonal shapes to be relevant for basal dislocation loops of intermediate size.

\section{Stability of loops upon MD ageing}

To better check the thermal stability of the dislocation loops, and thus establish the relevance of the former MS results, dislocation loops were annealed by $\mathrm{MD}$ at $573 \mathrm{~K}$ for $500 \mathrm{ps}$. Here, all loops are initially of circular shape, and are created by simple removal/addition of atoms. This is meant to prevent trapping in metastable states, as the initial loop configurations are not fully collapsed and can involve some high energy stacking. The formation energies obtained for the two EAM potentials are given in Figs. 5(a) and (b). The top views of prismatic and basal loops after the MD relaxations

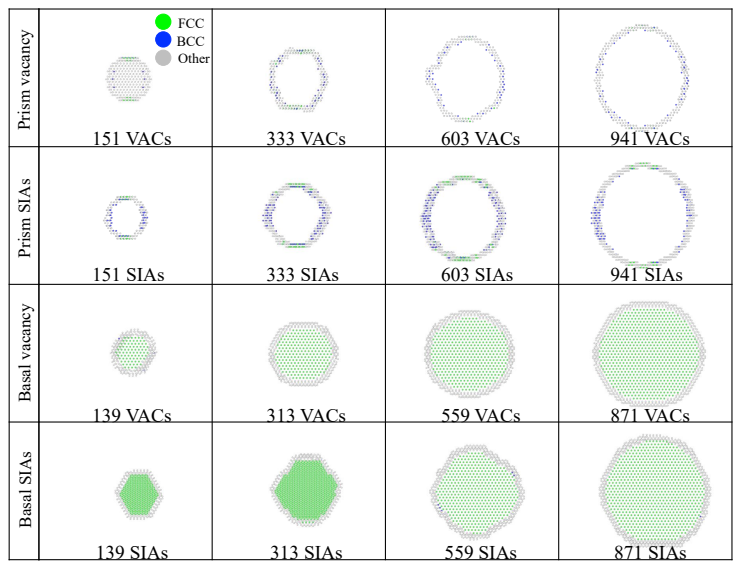

(a) EAM \#2

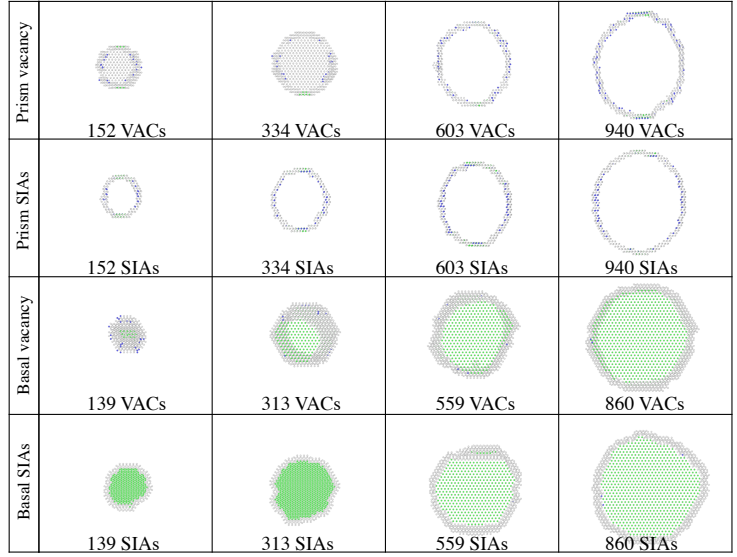

(b) EAM \#3

Figure 6: Top view of the loops after MD relaxation using the Zr (a) EAM \#2 and (b) \#3 potentials: prismatic vacancy and SIAs loops, and basal vacancy and SIAs loops. Atoms are colored according to CNA.

using the \#2 and \#3 potentials are illustrated in Figure 6(a) and (b), respectively. Atom coloring is based on the CNA.

The formation energies of vacancy and interstitial-type defects after MD ageing are in the same range as those obtained by MS simulations. The MD annealing allows the system to overcome some energy barriers and thus, for a given defect size and type (basal/prismatic, and vacancy/interstitial), we expect to observe the most stable structure, if the annealing is sufficiently long. The structural analysis of the defects indicate good consistency with the MS calculations in many aspects:

- Vacancy prismatic loops are faulted at small 
size and perfect at large sizes. Their interstitial counterparts are unfaulted.

- Some dissociation in both basal and pyramidal planes are seen for the prismatic loops, leading to a faceting especially at small/intermediate sizes and for interstitial loops, which are then more tilted with respect to the prismatic planes as compared to vacancy loops.

- Vacancy basal defects displays a $\mathrm{I}_{1}$ stacking fault for the whole range of defect sizes.

- SIAs basal loops have an E-fault at small sizes, and an $\mathrm{I}_{1}$ fault at larger size.

- The shapes of the basal loops evolves during the MD relaxation towards more hexagonal loops.

The above observations hold for both EAM potentials. On the other hand, some differences can be seen. First, the large prismatic loops with EAM \#3 potential imaged in the prismatic plane in Fig. 6(b) show a more elliptical shape, which is due partly to a slight tilt with respect to the prismatic plane during relaxation, and partly to a loop shape evolution. This shape agrees with experimental observations. Second, for the EAM \#3 potential, the smallest vacancy basal defect is a stacking fault pyramid, as described in Refs 29, 26. With increasing defect size, a progressive evolution towards $I_{1}$ faulted loop is observed. The potential energy landscape envisioned in the MS study, and as described by the EAM potentials used here, could then be completed by the addition of vacancy stacking fault pyramidtype of defect.

Note finally the small loop shape fluctuations seen in Fig 6 particularly visible for SIAs basal loops with EAM \#3. These could indicate that the equilibrium structures are not reached during the MD relaxations. To further check this, dislocation loops created in both hexagonal and circular shape using the BABEL package and as in MS calculations were also annealed by the same MD procedure as described previously. Formation energies and structural analysis are provided in Supplementary Information. While the MD results of Figs. 5 and 6 mostly provide more stable structures than the new MD calculations, some exceptions exists for hexagonal basal loops with EAM \#3. Given the rather high complexity of the loops configurations (SF, shape, tilting, etc.), an automated technique for potential energy landscape exploration would be necessary to ensure finding the equilibrium structures [57, 58, 59].

\section{Continuum modelling of dislocation loops energetics}

In this section we describe and make use of a continuum modelling for the formation energy of dislocation loops, having both fixed and adjustable parameters. Atomistic MS data for vacancy and interstitial loops, and for hexagonal or circular shapes, are fitted with such a model. This allows to identify the adjustable parameters and discuss their relevance. We finally re-parameterize the continuum laws with the fixed parameters coming from $a b i n i-$ tio calculations from the literature, and study the influence of the interatomic potential used for the adjustable parameter identification.

\subsection{Continuum elasticity laws for loops energetics}

The continuum model used here comes from Refs. [25, 27, 26] and captures the dislocation loop of radius $R$ 's formation energy $E_{f}$ using two components: a line tension elastic energy $E_{E}$ and a stacking fault energy contribution $E_{S}$, as

$$
\begin{aligned}
E_{f} & =E_{E}+E_{S}, \\
& =2 \pi R f \bar{K} \ln \left(\frac{R}{r_{\mathrm{c}}}\right)+\pi R^{2} \gamma .
\end{aligned}
$$

In this formula, $\bar{K}$ is the elastic coefficient of the loop, averaged assuming a circular shape and calculated within anisotropic elasticity [60, 61, 62] from the bulk elastic constants, the Burgers vector of the loop and the direction of the loop elements. $\gamma$ is the stacking fault energy of the loop. These two parameters are fixed parameters and are computed easily for a given interaction model for Zr; the quantities used to calculate them for the EAM potentials and from $a b$ initio can be found in Table 1. The remaining parameters, the shape factor $f$ and the effective core radius $r_{c}$ of the loop, are fitting parameters having a physical interpretation. The former accounts for two things: the real shape of the loop might not be circular, and the loop might experience a small glide along its glide cylinder during the atomistic relaxation. The latter adjustable parameter $r_{\mathrm{c}}$ can be related to the dislocation core energy as $r_{\mathrm{c}}=r_{0} \exp \left(-E_{\text {core }} / \bar{K}\right)$, 
where $r_{0}$ is the fixed core radius used for the determination of $E_{\text {core }}$. Note that the constant adjustable parameter reported in Refs. [26, 27] - that was meant to improve the quality of the fitting at small defect size (below $\simeq 50$ point defects) - is not considered in Eq. 4, as the atomic data of this study considers loops having more than 127 point defects. The link between the loop radius $R$ and the number of point defects constituting the various types of loops is given in Ref. 25].

This continuum model for the dislocation loop energetics is simple and includes a number of approximations, mostly in the loop elastic energy term that is described by the line tension. This neglects the elastic interactions between the dislocation line segments forming the loops, which might be important for large curvatures and thus small defect sizes. In addition, a unique effective core radius $r_{\mathrm{c}}$ - or core energy - is chosen, meaning that its orientation-dependence and defect size dependence are neglected. The core energy of large loops is usually assumed to be small compared to the strain elastic energy 63, 64, 65, but they may be comparable in the case of small dislocation loops, as emphasized by Kroupa [66]. The relevance of the simple modelling adopted here will be discussed later.

\subsection{Adjustment on $M S$ results}

The formation energies of dislocation loops from MS simulations are used to fit the unknown parameters $f$ and $r_{\mathrm{c}}$ in Eq. 4 . We perform the fitting separately for each EAM potential, and for hexagonal and circular loops. As analyzed in section 3 . the formation energies of those loops that changed their stacking sequence after the MS relaxation are not used during the fitting procedure. For SIAs prismatic faulted loops, that are partially or fully unstable for both potentials, the fitting is not performed. Two different fits were realized: one that includes the whole remaining atomistic datapoints, and one that excludes for each kind of loop the three datapoints corresponding to the smallest loop sizes. The motivation for this is that fewer variations with loop size in dislocation core structure and in misorientation are observed for loops having $\geq 300$ point defects. All obtained adjusted parameter values, as well as the $\bar{K}$ fixed parameter values are reported in Table 3 for the EAM \#2 and \#3 potentials. Figures in Supplementary Information shows the quality of the fits, which is good in all cases.

We first note that all fitted values for both $f$ and $r_{\mathrm{c}}$ are reasonable, i.e. of the order of magnitude of
1 for $f$ and of the order of an angstrom for $r_{\mathrm{c}}$, in agreement with their physical meaning. The adjusted parameters for vacancy loops with EAM \#2 can be compared with those already obtained in Ref. 25]. Our $\left(f, r_{\mathrm{c}}\right)$ values for the perfect prismatic and the $\mathrm{I}_{1}$-faulted basal vacancy loops are close to the previous ones, but differ for the faulted prismatic and E-faulted basal loops. The range of loop size for the fitted atomistic data is different in both works, e.g. the maximum loop size was 337 vacancies in Ref. [25] and extends over 1135 vacancies in the present work, which may explain the observed differences.

Looking now at the $r_{\mathrm{c}}$ values obtained for all type of loops from the fitting of the whole range of atomistic data, connections with the dislocation core structures analysed earlier can be examined. With EAM \#3, the effective core width is larger for SIA hexagonal perfect prismatic loops than for their vacancy counterparts, in agreement with atomistic observations (see Table 2). Values for vacancy and SIA loops with the EAM \#2 potential are closer to each other than with EAM \#3, similarly to atomistics; however $r_{\mathrm{c}}$ is greater for the vacancy loops, different from atomistics. Besides, $r_{\mathrm{c}}^{\# 3}<r_{\mathrm{c}}^{\# 2}$ for vacancy perfect prismatic loops, as in atomistics, but $r_{\mathrm{C}}^{\# 3}<r_{\mathrm{c}}^{\# 2}$ for the same defect of SIA type, which disagrees with atomistics. Next, for hexagonal basal loops, some correlation is found between $r_{\mathrm{c}}$ values and the analyzed dislocation core struc-

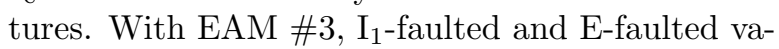
cancy loop segments are more dissociated in pyramidal planes than the interstitial loop segments, and they have higher $r_{\mathrm{c}}$ values. Core structures of basal vacancy and SIA loops are more similar in the case of the EAM \#2 potential, and accordingly they have similar $r_{\mathrm{c}}$. Besides, the adjusted effective core widths for the basal loops are smaller with the EAM \#2 than with the EAM \#3, and this again agrees with the atomistic observations (see Fig. (4). Now, if we consider the adjusted parameters for the reduced atomistic data range, we notice that their values are rather close to the previous ones for most defects. The only exception is for the E-faulted basal vacancy loop with the EAM \#3 potential: the adjusted $r_{\mathrm{c}}$ is seven times smaller when restricting the atomistic datapoints to the larger loop sizes, and thus becomes smaller than its SIA counterpart, contrary to what is seen in atomistic simulations. In this case, the physical meaning of $r_{\mathrm{c}}$ is clearly lost. Finally, we comment on the effective core radii obtained for circular loops. For 
Table 3: Parameters entering in the line tension model of Eq. 4 for the two Zr EAM potentials. The dimensionless shape factor $f$ and the effective core radius $r_{c}$ ( $a$ is lattice parameter) are adjusted parameters, obtained by separated fitting of the two sets of atomistic results. Values in italic font come from the fitting of a reduced dataset (see main text). The fixed elastic coefficients $\bar{K}$ (in eV/A) are taken from Ref [25] for EAM \#2, and calculated in this work for EAM \#3.

\begin{tabular}{|c|c|c|c|c|c|c|c|c|c|}
\hline \multirow[b]{2}{*}{ Loop } & & & & \multicolumn{3}{|c|}{ EAM \#2 } & \multicolumn{3}{|c|}{ EAM \#3 } \\
\hline & & & & $f$ & $r_{c} / a$ & $K$ & $f$ & $r_{c} / a$ & $K$ \\
\hline \multirow[t]{7}{*}{ hexagonal } & prism & perfect & $\mathrm{V}$ & 1.081 .09 & 0.230 .25 & 0.28 & 1.021 .01 & 0.190 .19 & 0.27 \\
\hline & & & $\mathrm{I}$ & 1.031 .05 & 0.180 .19 & 0.28 & 1.071 .10 & $0.34 \quad 0.38$ & 0.27 \\
\hline & & faulted & V & 1.701 .70 & $\begin{array}{lll}0.39 & 0.38\end{array}$ & 0.22 & 1.701 .70 & $\begin{array}{lll}0.62 & 0.61\end{array}$ & 0.23 \\
\hline & basal & $\mathrm{I}_{1}$ fault & $\mathrm{V}$ & $1.06 \quad 1.04$ & $\begin{array}{lll}0.25 & 0.23\end{array}$ & 0.33 & 1.061 .04 & $0.32 \quad 0.30$ & 0.30 \\
\hline & & & $\mathrm{I}$ & 1.081 .10 & $0.28 \quad 0.30$ & 0.33 & 0.950 .96 & $\begin{array}{lll}0.21 & 0.22\end{array}$ & 0.30 \\
\hline & & $\mathrm{E}$ fault & V & 1.151 .05 & 0.130 .09 & 0.24 & 1.620 .95 & $0.77 \quad 0.10$ & 0.22 \\
\hline & & & $\mathrm{I}$ & 1.091 .08 & $0.14 \quad 0.13$ & 0.24 & 1.051 .07 & 0.190 .20 & 0.22 \\
\hline \multirow[t]{7}{*}{ circular } & prism & perfect & $\mathrm{V}$ & 1.151 .25 & $0.28 \quad 0.39$ & 0.28 & 0.980 .96 & 0.170 .15 & 0.27 \\
\hline & & & $\mathrm{I}$ & 1.121 .21 & $0.24 \quad 0.32$ & 0.28 & 1.071 .10 & 0.330 .37 & 0.27 \\
\hline & & faulted & $\mathrm{V}$ & 1.701 .70 & 0.350 .33 & 0.22 & 1.701 .70 & 0.630 .63 & 0.23 \\
\hline & basal & $\mathrm{I}_{1}$ fault & $\mathrm{V}$ & 1.101 .10 & 0.280 .28 & 0.33 & 1.020 .93 & 0.230 .15 & 0.30 \\
\hline & & & I & 1.151 .17 & $0.34 \quad 0.37$ & 0.33 & $0.99 \quad 0.92$ & 0.230 .17 & 0.30 \\
\hline & & E fault & $\mathrm{V}$ & 1.141 .08 & 0.120 .09 & 0.24 & 1.100 .99 & 0.170 .10 & 0.22 \\
\hline & & & I & 1.151 .18 & $0.16 \quad 0.19$ & 0.24 & 0.990 .96 & 0.150 .13 & 0.22 \\
\hline
\end{tabular}

perfect prismatic loops, slightly smaller $r_{\mathrm{c}}$ values than those of hexagonal loops are obtained with the EAM \#3 potential, showing the same trend as in atomistics, although underestimated. For the same loops but with the EAM \#2 potential, $r_{\mathrm{c}}$ values are close for vacancy and SIA loops, and a bit larger than those of hexagonal loops, contrary to atomistic results. Concerning basal circular loops, the EAM \#3 potential shows $r_{\mathrm{c}}$ coefficients that are similar for vacancy and SIA types of loops, for both $\mathrm{I}_{1}$ and E-faulted defects. This similarity agrees with atomistics, but is less fulfilled for the EAM \#2 potential. Note that $r_{\mathrm{c}}$ adjusted values for circular loops are not very sensitive to the reference datapoints used for the fitting.

The shape factor $f$ of a dislocation loop is a bit harder to interpret. Its physical meaning is not limited to taking into account the difference in shape relative to a circle, but also include the effect of a possible small glide of the loop, and the fact that the elastic coefficient $\bar{K}$ is calculated for the line directions of a circular loop, and not for those of an hexagon, or of any kind of shape adopted by a loop after relaxation. $f$ values in Table 3 are essentially close to 1 , except for vacancy faulted prismatic loops with both potentials, and for vacancy E-faulted basal loop with EAM\#3 potential. The fitted shape factors of hexagonal loops are smaller (resp. larger) than those of circular loops with the EAM \#2 (resp. EAM \#3) potential. Contrary to what one could expect, the shape factors of circular loops are not closer to one than those of hexagonal loops. Atomistic simulations showed in general some misorientation and/or small shape evolution of the loops after relaxation; thus, deviations of $f$ from 1 can stem from this, as the shape and/or segment orientation deviate from those of the initial circle.

\subsection{Parameterization with ab initio data}

Based on the effective core radii $r_{c}$ and shape factors $f$ determined from the fitting of the empirical potential data, the continuum laws for the formation energy of dislocation loops (Eq. 4) can be reparameterized with the following ab initio quantities: the elastic coefficient $\bar{K}$ and stacking fault energies $\gamma$, taken from Ref. 25. The formation energies of basal and perfect prismatic dislocation loops, of vacancy and SIA type, and with up to 10000 point defects, are illustrated in Figure 7. These laws for the loop stabilities can typically be implemented into mesoscale models, e.g., to predict the kinetics of vacancy and SIA clustering in $\alpha$-Zr. In order to test the sensitivity of the continuum law predictions to the adjusted parameter values, we display in Figure 7(a-h) the continuum formation 
energies obtained with the $f$ and $r_{\mathrm{c}}$ coefficients for both EAM \#2 and \#3 potentials, for hexagonal and circular loops, and for the two considered atomistic data ranges.

Some similar trends can be seen across all plots. For all considered cases, and above $\sim 1000$ point defects, the most stable loops are perfect (or unfaulted) prismatic loops, followed by $\mathrm{I}_{1}$-faulted basal loops, and then by E-faulted basal loops. Energetic differences between vacancy and interstitial types of loops are smaller than differences between loops having different stacking sequences. SIA loop formation energies are lower or similar to those of vacancy loops. These general qualitative trends are thus not very sensitive to the potential, loop shapes, and atomistic data range tested in the present study.

However, looking at the formation energy curves in a more quantitative way, some significant differences can be observed. With the $f$ and $r_{\mathrm{c}}$ parameter values from the EAM \#3 potential (Fig. 7) (cd) $(\mathrm{g}-\mathrm{h}))$, the energetic differences between vacancy and SIA types of loops are larger than those obtained from the EAM \#2 potential (Fig. 7) (a-b)(ef)). They also lead to larger formation energies for the E-faulted basal loops than those obtained in the case of parameters from EAM \#2, while the $\mathrm{I}_{1}$ faulted basal loops and the perfect prismatic loops are less sensitive to the potential used to determine $f$ and $r_{\mathrm{c}}$. A stability inversion with increasing loop size between E-faulted loops and $\mathrm{I}_{1}$-faulted loops is seen in all cases, but the transition size is not the same, in particular when changing the potential: it is larger for parameters adjusted on EAM \#2 atomistic data. The curves obtained for the reduced atomistic dataset (Fig. 7(e-h)) show a general tendency to decrease the energetic differences between vacancy and SIA types of loops. Note finally that the effect of the loop shape, comparing the curves obtained for hexagonal loops (Fig. 7 (a,c,e,g)) to those for circular loops (Fig. $7(\mathrm{~b}, \mathrm{~d}, \mathrm{f}, \mathrm{h})$ ), is much more moderate.

Consequently, for a quantitative extrapolation at large size of the loop formation energies - in particular able to correctly discriminate vacancy from interstitial type of loops, giving the proper loop sizes for stability inversions and the right energy scales - both the chosen atomistic potential and the size range of the atomistic datapoints are influential factors.

\section{Discussion}

The MS atomistic calculations performed with the two selected interatomic potentials present qualitative consistency on loops' stability and structure. Vacancy and interstitial loops having the same Burgers vector show small to moderate formation energy differences, while more pronounced differences are seen for the core structure of the dislocation loop segments and the relaxed loop orientation. Hexagonal and circular loops of the same type have very small energetic differences, but their dislocation core structures substantially differ. This strong effect of the loop shape on the segment core structure was already underlined by de Diego et al. 30, 31. Hexagonal basal loops are generally more stable than circular loops for the studied loop sizes, based on MS and MD data of this study; examination of recent experiments [20, 18, suggests that $\langle c\rangle$-component loops could be hexagonal or faceted at sizes of a few tenth of nm, and circular for larger sizes. For prismatic loops, previous experiments widely reports loops circular or elliptical in shape [10, 17. Our MD annealing simulations indicate some faceting of the small loops, and more circular/elliptic shapes when increasing the loop size. Temperature, alloying element segregation to loops and external strain fields are factors that may affect the shape of observed loops.

From the present atomistic calculations and the continuum models parameterized with $a b$ initio quantities, one can make connections with the experimental observations mentioned at the beginning of this article. For perfect $\langle a\rangle$ loops, the $a b$ initio modelling with adjusted parameters from EAM \#3 (resp. EAM \#2), indicates that interstitial loops are slightly more stable (resp. equally stable) than vacancy loops. The vacancy/interstitial loop coexistence that is seen experimentally is thus supported by stability arguments. Their relative proportion is however temperature-dependent, with interstitial (vacancy) loops predominating at low (high) temperature. This could rather be related to the possible structural differences between vacancy and SIA prismatic loops, as suggested by our atomistic simulations (e.g., different dissociation distances and loop orientations). Indeed, the core structure of a loop is closely related to its mobility [55, 56, its ability to reorientate and thus its capture cross-section for diffusing point defects, and the way it reacts with any source of stress/strain. Thus the way vacancy and interstitial $\langle a\rangle$ loops 

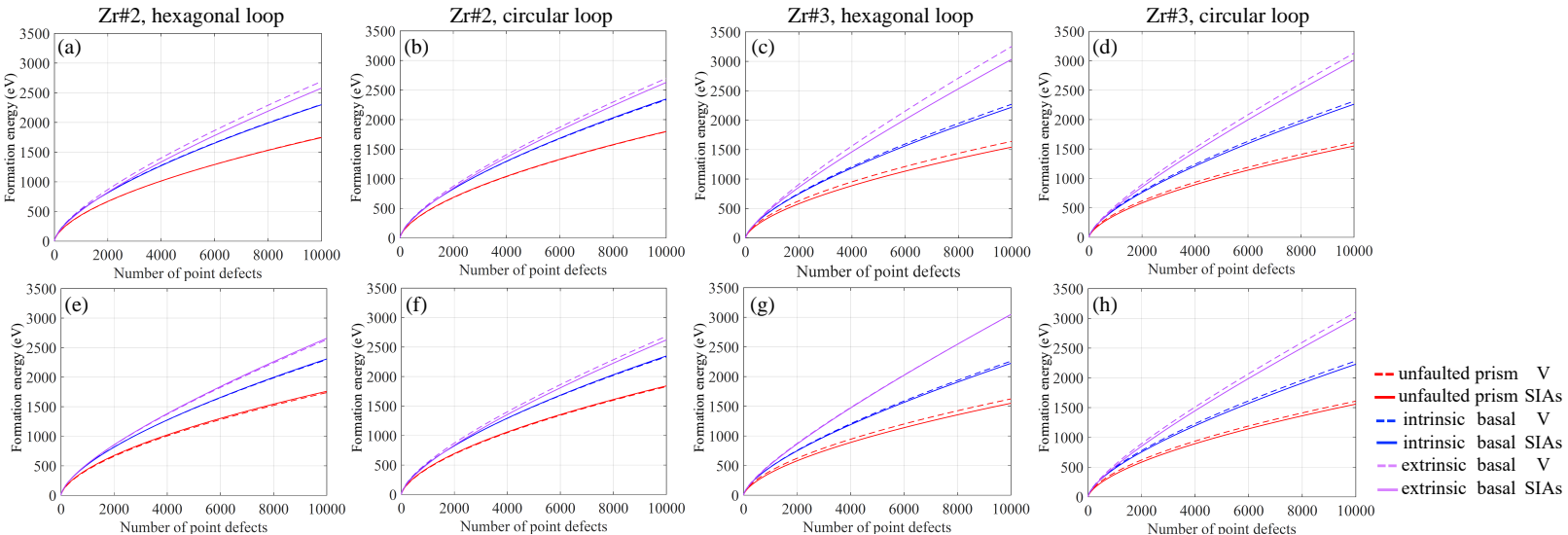

Figure 7: Formation energies of large loops predicted by continuum laws parameterized on DFT and empirical potential data for hexagonal loops ( $\mathrm{a}, \mathrm{c}$, e and $\mathrm{g}$ ) and circular loops (b, d, f and h) with the Zr \#2 (a, b, e and f) and \#3 (c, d, g and h) potentials. The reduced dataset are in (e-h).

grow and eliminate on the various sinks of the material might differ; this will be of importance for the ratio of observed vacancy/interstitial $\langle a\rangle$ loops on irradiated Zr alloys vs temperature. Recall that irradiation creep and growth are strongly controlled by the mobility and growth of loops 67. Considering now $\langle c\rangle$-component loops, the $a b$ initio modelling shows interstitial loops rather stabilized over vacancy E-faulted loops, and similar stability of vacancy and interstitial $\mathrm{I}_{1}$-faulted basal loops. This agrees with the possibility to observe both interstitial and vacancy basal loops in $\alpha$-Zr after electron irradiation [14. However, this cannot explain the fact that only vacancy-type $\langle c\rangle$ component loops are observed upon neutron irradiation [11, 12, which is most certainly related to the energetics and nucleation mechanisms of small clusters, as suggested in two recent works on vacancy basal clusters, showing stacking fault pyramids as possible precursors for basal loops [26, 29. To further clarify this, extensive $a b$ initio calculations on small SIAs clusters would be necessary. Another factor that could control the formation of basal loops is the diffusion of point defects and their clusters. The respective anisotropy of diffusion of vacancies and SIAs could cause a large bias in their reaction with sinks, and explain the widely observed formation of vacancy basal loops, as seen in the mesoscopic models of Refs. 21, 24. Also, Samolyuk et al. 68 included one-dimensional diffusion of cascade-induced SIA clusters in rate theory equations and reasonably explained low SIA cluster fluxes towards basal loops, which could fa- vor the growth of vacancy $\langle c\rangle$-component loops.

Another aspect highlighted in this work is the difficulty of obtaining quantitative continuum laws for the energetics of large loop sizes. The maximum loop size observed in experiments is about $150 \mathrm{~nm}$ [2, 13, 15, 10, 18, 19, which is equivalent to about 1 million point defects. This is beyond the available computational power to simulate such loop size using atomistic simulations. Thus, the usage of model's prediction at large size is of interest, but should be carried out with caution. In particular, we established that the $\left(f, r_{c}\right)$ adjusted values, and thus the $a b$ initio curves for the loops' formation energies, are sensitive to the choice of the potential and to the atomistic data range used for the fitting. As underlined earlier, the two EAM potentials used here have their own drawbacks for simulating irradiation defects in $\alpha-\mathrm{Zr}$. Consequently, this motivates the development of better force-fields, including the development of machine-learning based potentials, and also of order- $N$ Density Functional Theory methods for metals. In addition, our atomistic calculations show some dependencies that contradict the model's assumptions: the dislocation core structure (and thus $r_{\mathrm{c}}$ ) depends on the loop segment orientation, and on the defect size $n$ up to rather large sizes. This is very likely to remain, even with a better interaction model. The dependence in $n$ could possibly be avoided by a selfconsistent study of adjusted parameter values versus data range, which would require a large atomistic reference dataset. On the other hand, in order to get rid of the problem caused by the orientation- 
dependence of $r_{\mathrm{c}}$, and also by the shape factor $f$ that accounts for several physical effects, we could consider making the continuum model more complex. For instance, the initial loop shape could be explicitely included for the computation of the $\bar{K}$ coefficient, and the directional dependence of $r_{\mathrm{c}}$ included somehow. However, the atomistic calculations performed here indicate that the final loop shape, loop orientation and dislocation core structure evolve after relaxation, which might again present some difficulty for the adjustable parameters determination. Alternatively, one could keep the continuum models in their simple form and accompany their subsequent use in mesoscale models by a sensitivity analysis / uncertainty propagation study, clearly assuming that some of the continuum laws quantities are not well known. This would allow the determination of numerical boundaries, and to better support the predictions made.

\section{Conclusion}

The present work has unveiled formation energies of both vacancy and interstitial dislocation loops with all possible Burgers vectors/stacking sequences suggested by experiments in $\alpha$-Zr, using atomistic simulations based on two Zr EAM potentials and for defect size ranging from 127 to 1135 point defects. Then a line-tension model hybridly calibrated on atomistic results and $a b$ initio data allowed to predict formation energies at large defect sizes. In conclusion,

i) faulted prism SIAs loops were found essentially unstable after MS relaxation. For both $\mathrm{Zr}$ potentials, the stability of large loops are: perfect prism loop $>$ faulted prism loop $>$ intrinsic faulted basal loop > extrinsic faulted basal loop, as confirmed by MD annealings;

ii) the formation energy difference between vacancy and SIAs loops of the same type is moderate based on atomistics, and smaller for the \#2 potential. In general, SIA loops are more stable than vacancy loops for the investigated loop sizes;

iii) the hexagonal basal loops are more stable than the circular loops based on the atomistic data of this study, but differences are very small. MD annealing shows favourable faceting of prismatic loops for small loops; iv) the continuum models we provided for the loops' energetics can be used in mesoscale models for the defect microstructure evolution under irradiation environment. Their sensitivity to various factors indicate their limits for quantitative predictions.

A better understanding of formation sequence and evolution of dislocation loops would be helpful to estimate components' lifetime in nuclear reactors. However, the loop formation energy only provides information on thermodynamic aspects, while information from kinetic analysis would be necessary. In particular, a systematic exploration of the energetic landscape of the small point defect clusters ( 1 to 100 point defects) would address the question of the germination of the defects and possibly define more complex laws for the defect energetics 27. Another topic for future work would be a detailed study of the mobility mechanisms of large dislocation loops of vacancy and SIA character, which could show important differences, as suggest the structural differences identified in this work. Finally, energetics and kinetics of dislocation loops are affected by many other factors such alloying elements [17, 45] and other extended defects [16, 28, 43], that can induce elastic fields.

\section{Appendix A. MS Supercell size}

We performed convergence tests of the MS simulation box size. The prism and basal dislocation loops in hexagonal shape with 919 SIAs and 2791 SIAs were relaxed by MS simulations at zero stress, in system sizes ranged from 1.6 million to 6 million atoms, and their formation energies are shown in Figure A.8. Moreover, elastic corrections with the ANETO code 34 were applied, and consistency between the results was verified. The system size having 2.3 million atoms provide well-converged formation energies.

\section{Appendix B. Misorientation of the prism dislocation loops}

Measurements of the misorientation angles of the perfect prismatic loops after MS and MD relaxations were performed as a function of the loop size, and they are illustrated in Figures B.9(a) and (b). The given angles correspond to the angles between the initial and relaxed habit plane; the sign of angles follows the trigonometric usage. 

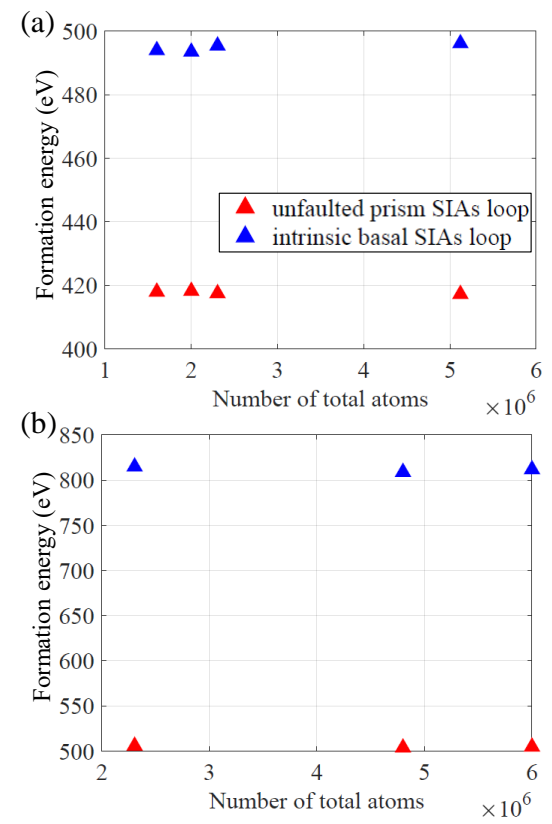

Figure A.8: Formation energy of SIAs dislocation loops by the $\mathrm{Zr} \# 2$ potentials. The loops are in hexagonal shape with 919 SIAs (a) and 2791 SIAs (b).

Note that in MD simulations (Figure B.9(b)), some annealed loops have changed more significantly of habit planes after relaxation. For example, some SIAs loops lie close to a first order prismatic plane different from the initial one, and some vacancy loops lie close to a second order prismatic plane. Those loop reorientation mechanisms observed during MD simulations are not commented here, this is a topic for future work.

\section{Appendix C. Dissociation distance}

Using anisotropic elastic theory of dislocations, the equilibrium dissociation distance of an infinite straight dislocation is given as

$$
d_{\mathrm{eq}}=\frac{b_{i}^{(1)} K_{i j} b_{j}^{(2)}}{\gamma},
$$

with $\mathbf{b}^{(\mathbf{1})}$ and $\mathbf{b}^{(\mathbf{2})}$ the Burgers vectors of the first and second partials, respectively. $\gamma$ is the relevant stable stacking fault energy, and $\mathbf{K}$ is the Stroh matrix [60, 61]. This quantity has been computed using the BABEL package [48].
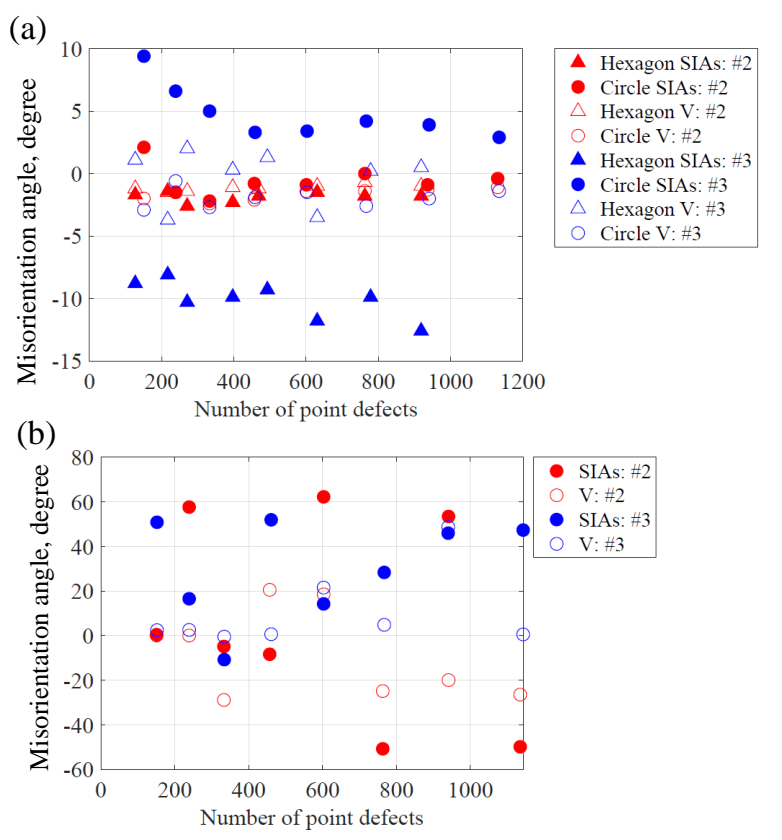

Figure B.9: The rotation angles of the habit plane for prism dislocation loops performed after MS (a) and MD (b) simulations by the Zr EAM \#2 and \#3 potentials.

\section{Acknowledgments}

The authors thank Compute Canada for generous allocation of computing resources. Computation for the work described in this paper was also supported by the Minerva High Performance Computing Cluster at Canadian Nuclear Laboratories. The research was supported by NSERC and the NSERC/UNENE Industrial Research Chair in Nuclear Materials at Queen's, and funded by Atomic Energy of Canada Limited, under the auspices of the Federal Nuclear Science and Technology Program. C. Varvenne thanks the French national agency ANR for funding this work through the grant project CoCoA ANR-CE08-002-0001, E. Clouet for fruitful discussions on dislocations and irradiation defects in hcp systems, and the H1 group at IPC-AMU for supporting discussions.

\section{References}

[1] T. D. Gulden, I. M. Bernstein, Dislocation loops in irradiated zirconium, Philos. Mag. 14 (131) (1966) 10871091. doi:10.1080/14786436608244779

[2] P. M. Kelly, R. G. Blake, The characterization of dislocation loops in neutron irradiated zirconium, Philos. Mag. 28 (2) (1973) 415-426. doi:10.1080/ 14786437308217463 
[3] F. Onimus, J. L. Béchade, 4.01 - radiation effects in zirconium alloys, in: E. in Chief: Rudy J.M. Konings (Ed.), Comprehensive Nuclear Materials, Elsevier, Oxford, 2012, pp. 1 - 31. doi:http://dx.doi.org/10. 1016/B978-0-08-056033-5.00064-1

[4] D. Northwood, V. Fidleris, R. Gilbert, G. J. C. Carpenter, Dislocation loop generation and irradiation growth in a zirconium single crystal, J. Nucl. Mater. 61 (2) (1976) 123 - 130. doi:https://doi.org/10. 1016/0022-3115(76)90076-3.

[5] G. Carpenter, R. Zee, A. Rogerson, Irradiation growth of zirconium single crystals: A review, J. Nucl. Mater. 159 (0) (1988) 86 - 100. doi:http://dx.doi.org/10. 1016/0022-3115(88)90087-6

[6] E. Ibrahim, R. Holt, Anisotropy of irradiation creep and growth of zirconium alloy pressure tubes, J. Nucl. Mater. 91 (2) (1980) 311 - 321. doi:https://doi.org/ 10.1016/0022-3115(80)90231-7.

[7] R. A. Holt, R. W. Gilbert, $\langle c\rangle$ - component dislocations in annealed zircaloy irradiated at about $570 \mathrm{k}$, J. Nucl. Mater. 137 (3) (1986) 185 - 189. doi:https: //doi.org/10.1016/0022-3115(86)90218-7.

[8] O. Mackain, M. Cottura, D. Rodney, E. Clouet, Atomicscale modeling of twinning disconnections in zirconium, Physical Review B 95 (13) (2017) 134102. doi:10.1103/ PhysRevB.95.134102

URL https://link.aps.org/doi/10.1103/PhysRevB. 95.134102

[9] R. Adamson, W. Bell, D. Lee, Use of ion bombardment to study irradiation damage in zirconium alloys, Zircon. Nucl. Appl. 551 (215-215-4). doi:doi:10.1520/ stp32117s

[10] A. Jostsons, P. M. Kelly, R. G. Blake, The nature of dislocation loops in neutron irradiated zirconium, J. Nucl. Mater. 66 (3) (1977) 236 - 256. doi:https: //doi.org/10.1016/0022-3115(77)90113-1.

[11] R. A. Holt, R. W. Gilbert, $\langle c>$-component dislocations in neutron irradiated zircaloy-2, J. Nucl. Mater. 116 (1) (1983) 127 - 130. doi:https://doi.org/10. 1016/0022-3115(83) 90301-X

[12] M. Griffiths, Microstructure evolution in h.c.p. metals during irradiation, Philos. Mag. A 63 (5) (1991) 835847. doi:10.1080/01418619108213917

[13] R. W. Gilbert, K. Farrell, C. E. Coleman, Damage structure in zirconium alloys neutron irradiated at 573 to $923 \mathrm{k}$, J. Nucl. Mater. 84 (1-2) (1979) 137-148. doi:10.1016/0022-3115(79)90157-0

[14] M. Griffiths, M. H. Loretto, R. E. Smallman, Anisotropic distribution of dislocation loops in hvemirradiated zr, Philos. Mag. A 49 (5) (1984) 613-624. doi:10.1080/01418618408233290

[15] D. Northwood, R. Gilbert, L. Bahen, P. Kelly, R. Blake, A. Jostsons, P. Madden, D. Faulkner, W. Bell, R. Adamson, Characterization of neutron irradiation damage in zirconium alloys - an international "roundrobin" experiment, J. Nucl. Mater. 79 (2) (1979) 379 394. doi:http://dx.doi.org/10.1016/0022-3115(79) 90103-X

[16] M. Griffiths, R. Gilbert, C. E. Coleman, Grain boundary sinks in neutron-irradiated zr and zr-alloys, J. Nucl. Mater. 159 (1988) 405-416. doi : https://doi.org/10. 1016/0022-3115(88)90107-9

[17] M. Griffiths, A review of microstructure evolution in zirconium alloys during irradiation, Journal of Nuclear Materials 159 (0) (1988) $190-218$. doi:http: //dx.doi.org/10.1016/0022-3115(88)90093-1. URL http://www.sciencedirect.com/science/ article/pii/0022311588900931

[18] A. Harte, D. Jädernäs, M. Topping, P. Frankel, C. P. Race, J. Romero, L. Hallstadius, E. C. Darby, M. Preuss, The effect of matrix chemistry on dislocation evolution in an irradiated zr alloy, Acta Mater. 130 (2017) 69 - 82. doi:https://doi.org/10.1016/j. actamat.2017.03.024

[19] M. Griffiths, R. Gilbert, V. Fidleris, R. Tucker, R. Adamson, Neutron damage in zirconium alloys irradiated at 644 to $710 \mathrm{k}$. Journal of Nuclear Materials 150 (2) (1987) 159 - 168. doi:http: //dx.doi.org/10.1016/0022-3115(87)90071-7, URL http://www.sciencedirect.com/science/ article/pii/0022311587900717

[20] G. Sundell, M. Thuvander, P. Tejland, M. Dahlbäck, L. Hallstadius, H. O. Andrén, Redistribution of alloying elements in Zircaloy-2 after in-reactor exposure, Journal of Nuclear Materialsdoi:10.1016/j.jnucmat.2014.07. 072

[21] C. H. Woo, Theory of irradiation deformation in non-cubic metals: Effects of anisotropic diffusion, Journal of Nuclear Materials 159 (C) (1988) 237-256. doi:10.1016/0022-3115(88)90096-7 URL http://www.sciencedirect.com/science/ article/pii/0022311588900967

[22] F. Christien, A. Barbu, Cluster dynamics modelling of irradiation growth of zirconium single crystals, J. Nucl. Mater. 393 (2009) 153-161. doi:10.1016/j.jnucmat. 2009.05 .016

[23] M. Christensen, W. Wolf, C. Freeman, E. Wimmer, R. Adamson, M. Griffiths, E. Mader, Vacancy loops in breakaway irradiation growth of zirconium: Insight from atomistic simulations, J. Nucl. Mater. 529 (2020) 151946. doi:https://doi.org/10.1016/j. jnucmat.2019.151946

[24] B. Christiaen, C. Domain, L. Thuinet, A. Ambard, A. Legris, Influence of vacancy diffusional anisotropy: Understanding the growth of zirconium alloys under irradiation and their microstructure evolution, Acta Materialia doi:10.1016/j.actamat.2020.06.004

[25] C. Varvenne, O. Mackain, E. Clouet, Vacancy clustering in zirconium: An atomic-scale study, Acta Mater. 78 (2014) 65-77. doi:10.1016/j.actamat.2014.06.012

[26] B. Christiaen, C. Domain, L. Thuinet, A. Ambard, A. Legris, A new scenario for $\langle c\rangle$-vacancy loop formation in zirconium based on atomic-scale modeling, Acta Mater. 179 (2019) 93 - 106. doi:https: //doi.org/10.1016/j.actamat.2019.07.030

[27] R. Alexander, M.-C. Marinica, L. Proville, F. Willaime, K. Arakawa, M. R. Gilbert, S. L. Dudarev, Ab initio scaling laws for the formation energy of nanosized interstitial defect clusters in iron, tungsten, and vanadium, Phys. Rev. B 94 (2016) 024103. doi:10.1103/ PhysRevB.94.024103

[28] M. I. Mendelev, G. J. Ackland, Development of an interatomic potential for the simulation of phase transformations in zirconium, Philos. Mag. Lett. 87 (2007) 349-359. doi:10.1080/09500830701191393

[29] C. Dai, P. Saidi, M. Topping, L. K. Béland, Z. Yao, M. R. Daymond, A mechanism for basal vacancy loop formation in zirconium, Scr. Mater. 172 (2019) 72 - 76. doi:https://doi.org/10.1016/j.scriptamat. 2019.07.006 
[30] N. de Diego, Y. N. Osetsky, D. J. Bacon, Structure and properties of vacancy and interstitial clusters in $\alpha$-zirconium, J. Nucl. Mater. 374 (2008) 87-94. doi: $10.1016 / j \cdot$ jnucmat.2007.07.011

[31] N. de Diego, A. Serra, D. J. Bacon, Y. N. Osetsky, On the structure and mobility of point defect clusters in alpha-zirconium: a comparison for two interatomic potential models, Modelling and Simulation in Materials Science and Engineering 19 (3) (2011) 035003.

URL http://stacks.iop.org/0965-0393/19/i=3/a= 035003

[32] G. J. Ackland, S. J. Wooding, D. J. Bacon, Defect, surface and displacement-threshold properties of $\alpha$-zirconium simulated with a many-body potential, Philos. Mag. A 71 (1995) 553-565. doi:10.1080/ 01418619508244468

[33] G. Vérité, C. Domain, C.-C. Fu, P. Gasca, A. Legris, F. Willaime, Self-interstitial defects in hexagonal close packed metals revisited: Evidence for low-symmetry configurations in Ti, Zr, and Hf, Phys. Rev. B 87 (2013) 134108. doi:10.1103/PhysRevB.87.134108

[34] C. Varvenne, F. Bruneval, M.-C. Marinica, E. Clouet, Point defect modeling in materials: coupling ab initio and elasticity approaches, Phys. Rev. B 88 (2013) 134102.

[35] G. D. Samolyuk, S. I. Golubov, Y. N. Osetsky, R. Stoller, Self-interstitial configurations in hcp zr: a first principles analysis, Phil. Mag. Lett. 93 (2) (2013) 93-100. doi:10.1080/09500839.2012.745653

[36] S. J. Wooding, L. M. Howe, F. Gao, A. F. Calder, D. J. Bacon, A molecular dynamics study of high-energy displacement cascades in $\alpha$-zirconium, Journal of Nuclear Materials doi:10.1016/S0022-3115(97)00365-6

[37] W. Zhou, J. Tian, J. Zheng, J. Xue, S. Peng, Dislocation-enhanced experimental-scale vacancy loop formation in hcp Zirconium in one single collision cascade, Scientific Reportsdoi:10.1038/srep21034

[38] Z. Wu, W. A. Curtin, Mechanism and energetics of $\langle c+a\rangle$ dislocation cross-slip in hcp metals Proceedings of the National Academy of Sciences 113 (40) (2016) 11137-11142. arXiv:https://www.pnas.org/ content/113/40/11137.full.pdf doi:10.1073/pnas. 1603966113

URL https : //www . pnas . org/content/113/40/11137

[39] N. Chaari, E. Clouet, D. Rodney, First-principles study of secondary slip in zirconium, Phys. Rev. Lett. 112 (2014) 075504. doi:10.1103/PhysRevLett.112.075504

[40] D. Rodney, L. Ventelon, E. Clouet, L. Pizzagalli, F. Willaime, $\mathrm{Ab}$ initio modeling of dislocation core properties in metals and semiconductors, Acta Mater. 124 (2017) 633 - 659. doi:http://dx.doi.org/10. 1016/j.actamat.2016.09.049.

[41] J. P. Perdew, K. Burke, M. Ernzerhof, Generalized gradient approximation made simple, Phys. Rev. Lett. 77 (1996) 3865-3868. doi:10.1103/PhysRevLett.77.3865 URL http://link.aps.org/doi/10.1103/ PhysRevLett.77.3865

[42] E. Clouet, Screw dislocation in zirconium: An $a b$ initio study Phys. Rev. B 86 (2012) 144104. doi:10.1103/PhysRevB.86.144104 URL http://link.aps.org/doi/10.1103/PhysRevB. 86.144104

[43] W. Szewc, L. Pizzagalli, S. Brochard, E. Clouet, Onset of plasticity in zirconium in relation with hydrides precipitation, Acta Mater. 114 (2016) 126 - 135. doi: https://doi.org/10.1016/j.actamat.2016.05.025

[44] B. Yin, Z. Wu, W. A. Curtin, Comprehensive firstprinciples study of stable stacking faults in hcp metals Acta Mater. 123 (2017) 223 - 234. doi:https: //doi.org/10.1016/j.actamat.2016.10.042 URL $\mathrm{Zr}$

[45] P. Villars, L. D. Calvert, Pearson's handbook of crystallographic data for intermetallic phases, Am. Soc. Metals, Materials Park, OH, USA, 1985.

[46] E. S. Fisher, C. J. Renken, Single-crystal moduli and the hcp $\rightarrow$ bcc transformation in Ti, Zr and Hf, Phys. Rev. 135 (2A) (1964) 482-494.

[47] S. Plimpton, Fast parallel algorithms for short-range molecular dynamics, J. Comput. Phys. 117 (1) (1995) 1 - 19. doi:http://dx.doi.org/10.1006/jcph.1995. 1039

[48] E. Clouet, Babel package (2018).

URL doi:http://emmanuel.clouet.free.fr/ Programs/Babel/index.html

[49] J. P. Hirth, J. Lothe, Theory of Dislocations, 2nd Edition, Wiley, New York, 1982.

[50] C. Dai, L. Balogh, Z. Yao, M. R. Daymond, Atomistic simulations of the formation of $\langle c\rangle$-component dislocation loops in $\alpha$-zirconium, J. Nucl. Mater. 478 (2016) 125 - 134. doi:https://doi.org/10.1016/j.jnucmat. 2016.06 .002

[51] A. Stukowski, Visualization and analysis of atomistic simulation data with ovito-the open visualization tool Modelling Simul. Mater. Sci. Eng. 18 (1) (2010) 015012 .

URL http://stacks.iop.org/0965-0393/18/i=1/a= 015012

[52] D. Faken, H. Jónsson, Systematic analysis of local atomic structure combined with 3d computer graphics, Comput. Mater. Sci. 2 (2) (1994) $279-286$. doi : https : //doi.org/10.1016/0927-0256(94)90109-0

[53] A. Stukowski, K. Albe, Extracting dislocations and non-dislocation crystal defects from atomistic simulation data, Modell. Simul. Mater. Sci. Eng. 18 (8) (2010) 085001. doi:10.1088/0965-0393/18/8/085001

[54] D. Hull, D. J. Bacon, Introduction to Dislocations, 5th Edition, Butterworth-Heinemann, Oxford, UK, 2011.

[55] M. S. Duesbery, The influence of core structure on dislocation mobility, Philosophical Magazinedoi:10.1080/ 14786436908216308

[56] W. Cai, V. V. Bulatov, J. Chang, J. Li, S. Yip, Chapter 64 Dislocation core effects on mobility, in: Dislocations in Solids, 2004. doi:10.1016/S1572-4859(05)80003-8.

[57] A. F. Voter, F. Montalenti, T. C. Germann, Extending the time scale in atomistic simulation of materials, Annual review of materials research 32 (1) (2002) 321-346.

[58] L. K. Béland, P. Brommer, F. El-Mellouhi, J.-F. Joly, N. Mousseau, Kinetic activation-relaxation technique, Physical Review E 84 (4) (2011) 46704.

[59] L. K. Béland, Y. N. Osetsky, R. E. Stoller, H. Xu, Kinetic activation-relaxation technique and selfevolving atomistic kinetic Monte Carlo: Comparison of on-the-fly kinetic Monte Carlo algorithms, Computational Materials Science 100 (PB) (2015) 124-134. doi:10.1016/j.commatsci.2014.12.001 URL http://www.sciencedirect.com/science/ article/pii/S0927025614008386

[60] A. N. Stroh, Dislocations and cracks in anisotropic elasticity, Philos. Mag. 3 (1958) 625-646. doi:10.1080/ 14786435808565804 
[61] A. N. Stroh, Steady state problems in anisotropic elasticity, J. Math. Phys. (Cambridge, Mass.) 41 (1962) 77.

[62] E. Clouet, Elastic energy of a straight dislocation and contribution from core tractions, Philosophical Magazine 89 (19) (2009) 1565-1584. doi:10.1080/ 14786430902976794

URL https : //doi.org/10.1080/14786430902976794

[63] R. Bullough, E. A. J. Foreman, The elastic energy of a rhombus-shaped dislocation loop, Philos. Mag. 9 (1964) 315-329. doi:10.1080/14786436408229194

[64] D. J. Bacon, The elastic energies of elliptical dislocation loops, Philos. Mag. A 14 (130) (1966) 715-729. arXiv: https://doi.org/10.1080/14786436608211967, doi: 10.1080/14786436608211967

[65] R. Dewit, A. W. J. Ruff, The elastic energies of non-regular hexagonal dislocation loops, Philos. Mag. A 15 (137) (1967) 1065-1069. doi:10.1080/ 14786436708221654

[66] F. Kroupa, Circular edge dislocation loop, Czechoslov. J. Phys. B 10 (4) (1960) 284-193. doi:10.1007/ BF02033533.

[67] R. A. Holt, Microstructure dependence of irradiation creep and growth of zirconium alloys, Journal of $\mathrm{Nu}-$ clear Materials 90 (1-3) (1980) 193-204.

[68] G. D. Samolyuk, A. V. Barashev, S. I. Golubov, Y. N. Osetsky, R. E. Stoller, Analysis of the anisotropy of point defect diffusion in hcp $\mathrm{Zr}$. Acta Materialia 78 (2014) 173-180. doi:10.1016/j .actamat.2014.06.024 URL http://www.sciencedirect.com/science/ article/pii/S1359645414004443 